\title{
Metformin enhances osteogenic differentiation of stem cells from human exfoliated deciduous teeth through AMPK pathway
}

\section{Xuedan Zhao}

Key Laboratory of Oral Medicine, Guangzhou Institute of Oral Disease, Affiliated Stomatology Hospital of Guangzhou Medical University, Guangzhou, China 510140

\section{Wenyan Huang}

Key Laboratory of Oral Medicine, Guangzhou Institute of Oral disease, Affiliated Stomatology Hospital of Guangzhou Medical University, Guangzhou, China 510140

\section{Janak L Pathak}

Key Laboratory of Oral Medicine, Guangzhou Institute of Oral Disease, Affiliated Stomatolgy Hospital of Guangzhou Medical University, Guangzhou Medical University, Guangzhou, China 510140

\section{Chuandong Zhu}

Key Laboratory of Oral Medicine, Guangzhou Institute of Oral Disease, Affiliated Stomatology Hospital of Guangzhou Medical University, Guangzhou 510140, China

\section{Yunyang Li}

Key Laboratory of Oral Medicine, Guangzhou Institute of Oral Disease, Affiliated Stomatology Hospital of Guangzhou Medical University, Guangzhou 510140, China

\section{Hongbing Guan}

Key Laboratory of Oral Medicine, Guangzhou Institute of Oral Disease, Affiliated Stomatology Hospital of Guangzhou Medical University, Guangzhou 510140, China

\section{Yan Shu ( $\nabla$ yshu@rx.umaryland.edu )}

key laboratory of oral medical,guangzhou institute of oral disease,affiliated stomatology hospital of guangzhou medical university,guangzhou,china510140

\section{Sujuan Zeng}

Key Laboratory of Oral Medicine, Guangzhou Institute of Oral Disease, Affiliated Stomatology Hospital of Guangzhou Medical University, Guangzhou 510140, China

\section{Linhu Ge}

Key Laboratory of Oral Medicine, Guangzhou Institute of Oral Disease, Affiliated Stomatology Hospital of Guangzhou Medical University, Guangzhou 510140, China

\section{Research}

Keywords: Metformin, SHEDs, Tissue engineering, Osteogenesis, AMPK signaling 
Posted Date: April 7th, 2020

DOI: https://doi.org/10.21203/rs.3.rs-21218/v1

License: (c) (1) This work is licensed under a Creative Commons Attribution 4.0 International License. Read Full License

Version of Record: A version of this preprint was published at Journal of Tissue Engineering and Regenerative Medicine on October 26th, 2020. See the published version at https://doi.org/10.1002/term.3142. 


\section{Abstract}

Stem cells from human exfoliated deciduous teeth (SHEDs) are ideal seed cells in bone tissue engineering. As a first-line anti-diabetic drug, metformin has recently been found to promote bone formation. The purpose of this study was to investigate the effect of metformin on osteogenic differentiation of SHEDs and its underlying mechanism. SHEDs were isolated from the dental pulp of deciduous teeth from healthy children aged from 6 to 12, and their surface antigen markers of stem cells were detected by flow cytometry. The effect of metformin $(10-200 \mu \mathrm{M})$ treatment on SHEDs cell viability, proliferation, and osteogenic differentiation was analyzed. The activation of adenosine 5'monophosphate-activated protein kinase (AMPK) was determined by western blot assay for the AMPK phosphorylated at Thr172 (p-AMPK). SHEDs were confirmed as mesenchymal stem cells (MSCs) based on the expression of characteristic surface antigens. Metformin $(10-200 \mu \mathrm{M})$ did not affect the viability and proliferation of SHEDs, but significantly increased the expression of osteogenic genes, the activity of alkaline phosphatase, matrix mineralization, and p-AMPK level in SHEDs. Compound C, a specific inhibitor of AMPK pathway, abolished metformin-induced osteogenic differentiation of SHEDs. Moreover, metformin treatment enhanced pro-angiogenic/osteogenic growth factors BMP2 and VEGF but reduced the osteoclastogenic factor RANKL/OPG expression in SHEDs. In conclusion, metformin could induce the osteogenic differentiation of SHEDs by activating the AMPK pathway and regulates the expression of pro-angiogenic/osteogenic growth factors and osteoclastogenic factors in SHEDs. Therefore, SHEDs, combined with metformin possesses therapeutic potential for bone regeneration and bone defect repair.

\section{Introduction}

Effective reconstruction of the severe bone defects caused by trauma, infection, and tumor resection is a great challenge in clinical practice. Grafting autologous bone or synthetic bone substitutes are used to fill the large size bone defect. Bone grafts are the second most common tissue grafts in the world. Approximately 2,200,000 bone grafts are performed worldwide each year to repair bone defects [1-3]. However, supplies of functional stem cells and growth factors are necessary to regenerate the de novo bone. Therefore, development of functional stem cells with osteogenic potential is urgent to achieve ideal bone regeneration and repair.

Recently, the application of stem cells to bone regeneration and repair has been paid much attention. Stem cells from human exfoliated deciduous teeth (SHEDs) are characterized as mesenchymal stem cells (MSCs) with low immunogenicity. SHEDs can be differentiated into osteoblasts, odontoblasts, chondrocytes, adipocytes, nerve cells, and other cells with strong horizontal differentiation capability [4]. The potential clinical application of SHEDs is not only limited to dental diseases but also to a variety of disorders, including bone defects that may be repaired by tissue engineering with stem cells [5]. The telomere length of SHEDs is twice that of the human dental pulp stem cells (DPSCs), resulting in a high degree of stemness and vitality for SHEDs [6]. Compared with DPSCs and BMSCs, SHEDs have stronger proliferative, angiogenesis, and osteogenic potential $[7,8]$. In addition, SHEDs are conveniently available from dental practice with relatively low ethical barriers. SHEDs recovered after long-term cryopreservation 
retained stem cell characteristics, pluripotency, dentin/bone regeneration, and immunomodulatory function [9], this indicates the possibility of using cryopreserved autologous SHEDs for bone tissue engineering. SHEDs have thus been increasingly recognized as one of the most promising seed cell sources in tissue engineering for bone regeneration and repair.

Previous studies have shown that type 2 diabetes may cause abnormal bone formation $[10,11]$. Metformin is a safe, well-tolerated, and the first-line drug for the treatment of type 2 diabetes. and has benn approved for clinical use in the United Kingdom since 1958 and the United States since 1995 [12, 13]. In recent years, metformin has been found to promote MSCs to differentiate into osteoblasts [11, 14], as well as improve bone repair in diabetic patients $[11,15]$. As a highly hydrophilic compound, metformin requires membrane transporters such as organic cation transporters (OCTs) to cross the cell membrane and function [16]. OCTs have been found to be present in SHEDs[17], indicating metformin may exert an

effect on SHEDs. However, the effect of metformin on the osteogenic differentiation of SHEDs has not yet to be characterized.

Adenosine 5'-monophosphate-activated protein kinase (AMPK) is a crucial cellular energy sensor associated with cell viability [18]. Metformin has been well characterized as an activator of AMPK. The promotion of osteogenic differentiation of MSCs and bone repair in diabetic patients by metformin has led to establishing a role of AMPK in bone metabolism. The purpose of this study was to investigate whether metformin could promote the osteogenic differentiation of SHEDs and if so, to further evaluate the role of AMPK pathway in metformin-mediated osteogenic effect on SHEDs.

\section{Material And Methods}

\section{Cell Culture}

The Medical Ethics Committee of the Affiliated Stomatology Hospital of Guangzhou Medical University approved this study (KY2019008). Retained deciduous teeth were extracted from healthy children aged 6-12 years. The informed written consents from the parent of the patients was obtained. The teeth were cleaned in sterile conditions with phosphate buffered saline (PBS) containing $4 \%$ penicillin/streptomycin. Then pulp tissues were separated, cut into pieces, digested with $3 \mathrm{~g} / \mathrm{L}$ collagenase I and $4 \mathrm{~g} / \mathrm{L}$ dispase for $30 \mathrm{~min}$, and centrifuged for $5 \mathrm{~min}$. The collected cells were cultured in $60 \mathrm{~mm}$ petri dishes with a-MEM containing $10 \%$ of fetal bovine serum and $1 \%$ of penicillin/streptomycin. When the monolayer of adherent cells reached $80 \%$ of confluence, they were trypsinized and subcultured at $5 \times 10^{3} \mathrm{cells} / \mathrm{cm}^{2}$. Surface antigens (CD105, CD90, CD73, CD34, CD45) were detected by flow cytometry (FCM). CD73 and CD105 were labeled by Phcoerythrin (PE). CD90 and CD45 were labeled by Phycoerhthrin-CY5 Conjugate (PECY5). CD34 was labeled by Fluorescein isothiocyanate (FITC). The SHEDs of passage (P) 4 to 6 (P4-P6) were used for subsequent experiments.

\section{Cell Viability Staining}


SHEDs were seeded in 6-well plates at a density of $5 \times 10^{4}$ cells/well. The plates were put in a humidified incubator at $37^{\circ} \mathrm{C}$ under $5 \% \mathrm{CO}_{2}$ for $24 \mathrm{~h}$. After were starved for 12 hours on the culture medium containing $1 \%$ FBS. SHEDs were co-cultured with the DMEM medium containing $0,10,50,100$, and $200 \mu \mathrm{M}$ metformin (Sigma Aldrich, USA) was then added respectively. At day 1 and 7 , the cells were stained with a live/dead cell double staining kit (BestBio, China). The living cells were visualized as green and the dead cells as red under the inverted fluorescence microscopy (Leica Instruments, Houston, USA) according to the kit instruction.

\section{Cell Proliferation Assay}

Cells were cultured in 96-well plates at a density of $2 \times 10^{3}$ cells/well for $24 \mathrm{~h}$. Then SHEDs were cocultured with the medium containing $0,10,50,100$, and $200 \mu \mathrm{M}$ metformin was added respectively (6 repeats per concentration). At 1, 3, 5, or 7 days, the medium was discarded and the cells were washed

with PBS twice. The cells were then incubated for $2 \mathrm{~h}$ in the incubator after added $90 \mu \mathrm{l}$ medium and $10 \mu \mathrm{l}$ CCK8 reagent (Dojindo, Japan) to each well and the absorbance was measured at $450 \mathrm{~nm}$. The absorbance value of each time point was calculated, with the detection time as the abscissa and the absorbance value as the ordinate, and the histogram and line graph were drawn for statistical analysis. Each assay was repeated in triplicate $(n=3)$.

\section{Osteogenic Gene Expression Analysis}

SHEDs $\left(1.0 \times 10^{5}\right.$ cells) were seeded in each well of 6 -well plates. At 4 and 7 days after induction for SHEDs by metformin, total RNA was extracted using Trizol ${ }^{\circledR}$ (Invitrogen, Carlsbad, CA), and 1,000 ng of total RNA was used for the RT reaction using the PrimeScript RT reagent kit(Takara). qRT-PCR was performed using SYBR Green PCR Master Mix (Thermo Fisher, USA) to detect the gene expression of runtrelated transcription factor 2 (Runx2), type I collagen (COL-I), alkaline phosphatase (ALP), osteocalcin $(\mathrm{OCN})$, bone morphogenetic protein 2 (BMP2), vascular endothelial growth factor A (VEGFA), receptor activator of nuclear factor-KB ligand (RANKL), and osteoprotegerin (OPG). The primer sequences for the genes are listed in Table 1. PCR conditions were as follows: $2 \mathrm{~min}$ at $50^{\circ} \mathrm{C}, 10 \mathrm{~min}$ at $95^{\circ} \mathrm{C}$, then $15 \mathrm{sec}$ at $95^{\circ} \mathrm{C}$ for 40 cycles, and $60^{\circ} \mathrm{C}$ for 1 min in 96-well plates using the ViiA 7 q 7 -PCR System. The data were normalized to the internal control, GAPDH. The final expression level of the gene of interest relative to controls was reported by the $2-\Delta \Delta C$ t method. All experiments were repeated in triplicate $(n=3)$. 
Table 1

Primers used in qRT-PCR to examine gene expression.

\begin{tabular}{|lll|}
\hline Genes & Primer Sequence & \\
\cline { 2 - 3 } & $\begin{array}{l}\text { Forward } \\
\mathbf{5}^{\prime} \mathbf{3}^{\prime}\end{array}$ & $\begin{array}{l}\text { Reverse } \\
\mathbf{5}^{\prime} \mathbf{3}^{\prime}\end{array}$ \\
\hline ALP & GGACCATTCCCACGTCTTCAC & CCTTGTAGCCAGGCCCATTG \\
OCN & TGCCTGGAGAGGAGCAGAACT & GGCGCTACCTGTATCAATGGC \\
COL-I & CAGTGGTAGGTGATGTTCTGGGAG & CAAGAGGCATGTCTGGTTCGG \\
RUNX2 & CCCGTGGCCTTCAAGGT & CGTTACCCGCCATGACAGTA \\
GAPDH & GGACCTGACCTGCCGTCTAG & GTAGCCCAGGATGCCCTTGA \\
\hline VEGF-A & AGGAGGAGGGCAGAATCATCA & CTCGATTGGATGGCAGTAGCT \\
\hline OPG & CACTACTACACAGACAGCTGG & ACTCTATCTCAAGGTAGCGCC \\
\hline RANKL & CGTTGGATCACAGCACATCAG & GTACCAAGAGGACAGACTCAC \\
\hline BMP2 & AACACTGTGCGCAGCTTCC & CTCCGGGTTGTTTTCCCAC \\
\hline
\end{tabular}

\section{Western Blotting}

Total protein from SHEDs was extracted with RIPA buffer. The lysate was centrifuged at 12,000 rpm for 15 min to collect the supernatant. A bicinchoninic acid (BCA)-based protein analysis kit (BestBio, China) was used to quantify the protein concentration of lysate at $562 \mathrm{~nm}$. Then $30 \mu \mathrm{g}$ protein was isolated in $10 \%$ SDS-PAGE gel and transferred into polyvinylidene fluoride (PVDF) membrane. The membrane was blocked with $5 \%$ nonfat milk powder for $1 \mathrm{~h}$ at $37^{\circ} \mathrm{C}$. The membrane was then incubated at $4{ }^{\circ} \mathrm{C}$ overnight with primary antibodies, which included rabbit anti-human AMPKa, rabbit anti-human p-AMPK (Thr172), and rabbit anti-human GAPDH (Cell Signaling Technology, USA). HRP-conjugated Affinipure Goat Anti-Rabbit IgG was incubated at room temperature for $1 \mathrm{~h}$. Protein bands were detected by using an Enhanced Chemical Luminescence kit (Millipore, USA). The ImageJ software was used to semi-quantify band intensity.

\section{Alkaline Phosphatase (alp) Activity And Staining}

The cells $\left(1.0 \times 10^{4}\right.$ cells) were inoculated in each well of 48 -well plates, and $250 \mu$ l complete medium was added to each well. After $24 \mathrm{~h}$, different concentrations of metformin were added to the medium. On day 4 , the original medium was removed, and the cells were washed with PBS twice. The cell lysis buffer containing $0.1 \%$ Triton $x-100$ was added to each well. The cells were lysed on ice for 30 min and 
transferred into $1.8 \mathrm{ml}$ tubes, which were centrifuged $(12,000 \mathrm{rpm})$ at $4{ }^{\circ} \mathrm{C}$ for $15 \mathrm{~min}$. The supernatant is absorbed for subsequent operation. The protein concentration was determined by the BCA method, as described above. The activity of alkaline phosphatase was determined by alkaline phosphatase assay kit (Nanjing Jiancheng, China), according to the manufacturer's instruction. The experiment was replicated three times $(n=3)$.

For ALP staining, the cells were washed twice with PBS. The cells were then fixed with $150 \mu \mathrm{l}$ of alcohol (95\%) for $15 \mathrm{~min}$. The staining buffer was prepared by mixing $3 \mathrm{ml}$ of alkaline phosphatase color buffer, $10 \mu$ l of 5-bromo-4-chloro-3-indolyl phosphate (BCIP) solution, and $20 \mu \mathrm{l}$ of nitrotetrazolium blue chloride (NBT) solution in a $5 \mathrm{ml}$ tube according to the instructions of an alkaline phosphatase staining kit (Beyotime, China). The BCIP/NBT staining solution (150 $\mu$ l) was added to each well. After incubation in the dark at room temperature for $30 \mathrm{~min}$ for color development, the BCIP/NBT staining solution was removed. The cells were then washed with deionized water twice to terminate a color development reaction. The staining was examined under a stereomicroscope (Leica Instruments, Houston, USA)

\section{Mineralization Assays}

SHEDs were seeded into 48 -well plates with a density of $2 \times 10^{4}$ cells/well. After $24 \mathrm{~h}$, the cell culture medium was replaced with osteogenic medium $(50 \mathrm{~g} / \mathrm{ml}$ ascorbic acid, $10 \mathrm{mM}$ sodium glycerolphosphorate, $10 \mathrm{nM}$ dexamethasone) containing different concentrations of metformin. The medium was changed every 2 days. On days 14 and 21, the medium was discarded. The cells were fixed with $150 \mu$ l of ethanol (95\%) for $30 \mathrm{~min}$. Each well was then washed with double distilled water for 3 times. The cells were incubated with $5 \%$ of alizarin red (Sigma Aldrich, USA) dye solution for 5-10 min, and washed with double distilled water repeatedly until the washing water became colorless. The plate was placed under a stereomicroscope (Leica Instruments, Houston, USA) for observation and photo taking. After taking photos, each well of cells was incubated with $150 \mu \mathrm{l} 10 \%$ of the CPC solution for $1 \mathrm{~h}$ to extract the dye. The eluted liquid was transferred to the 96-well plate. The absorbance was measured by the micro plate spectrophotometer (Thermo Fisher, USA) at a wavelength of $562 \mathrm{~nm}$.

\section{Statistical analysis}

Data were obtained from at least three separate experiments under identical conditions and expressed as the mean \pm standard deviation (SD). Statistical analysis was performed by analysis of variance (ANOVA) and then Dunnett's test with the Graph Pad Prism software (Version 7, La Jolla, CA, USA). $P<0.05$ was considered statistically significant.

\section{Results}

\section{Stem Cell Surface Markers in Primary SHEDs}


Flow cytometry was used to examine the surface markers of MSCs in the SHEDs we isolated from healthy children aged 6-12 years. Consistent with other MSCs, the SHEDs showed a strong expression of mesenchymal surface molecular markers of the cluster of differentiation (CD), including CD105, CD90, and CD73 (Fig. 1). Besides, SHEDs expressed CD34 and CD45 moderately. These results confirm that the SHEDs isolated and used in this study are typical MSCs.

\section{No Effect Of Metformin Treatment On Sheds Viability}

To ascertain whether metformin treatment would have an impact on the growth of SHEDs, we compared the cell viability and proliferation between with and without metformin treatment. Figure 2a shows live/dead cell staining at days 1 and 7. The majority of cells were alive (green) under metformin treatment $(10-200 \mu \mathrm{M})$ with a few dead cells (red). As shown by quantitative analysis, metformin treatment did not affect SHEDs viability (Fig. 2b), nor the cell growth over time up to 7 days (Fig. 2c). No adverse effect of metformin treatment on SHEDs proliferation and growth over the time course of induction (1-7 days) was further confirmed by another viability/proliferation assay (CCK8) (Fig. 2d).

\section{Effect of Metformin Treatment on Osteogenic Gene Expression of SHEDs}

To study the impact of metformin treatment on osteogenic differentiation of SHEDs, we first examined the effects on the expression of related osteogenic genes. q-PCR was performed at day 4 and 7 after metformin treatment. VEGFA, a member of the platelet-derived growth factor (PDGF)/VEGF growth factor family, plays a vital role in angiogenesis [19]. BMP is an acidic glycoprotein widely existing in the bone matrix, and BMP2 is one of the key extracellular signaling molecules promoting bone formation and inducing osteoblast differentiation [20]. OCN is considered as a marker of osteoblast differentiation and maturation [21]. Runx2 is an important regulator of osteoblast differentiation. ALP and COL-I are also well known as makers of bone formation. Compared to the control, the cells treated with metformin had significantly increased expression of ALP, RUNX2, COL-I, OCN, BMP2, and VEGFA at days 4 and 7 (Fig. 3af).

Furthermore, bone healing involves synthesis by osteoblast and bone resorption by osteoclast and is tightly controlled by the RANKL/RANK/OPG molecular triplet. RANKL/OPG is the key to maintain the dynamic balance between bone resorption and bone formation in bone remodeling [22]. Consistent with the above upregulation of genes in bone formation, the expression of RANKL/OPG in metformin-treated cells was significantly lower than that in vehicle-treated control cells (Fig. $3 g$ ).

\section{Effects Of Metformin Treatment On Mineralization In Sheds}


To further assess the effects of metformin treatment on osteogenic differentiation of SHEDs, we examined matrix mineralization of SHEDs culture by using Alizarin Red staining. ARS was performed at days 14 and 21 after osteogenic induction. The dense staining in Fig. 4a indicates the higher degree of matrix mineralization in the metformin-treated SHEDs than those in the control cells at day 14 (Fig. 4a, left panels). By quantitative analysis on the dye extract with $10 \% \mathrm{CPC}$, we found that metformin treatment for 14 days significantly increased the mineralization in SHEDs as compared to the vehicletreated controls (Fig. 4b). However, there were no differences between metformin treatment and vehicle at day 21 (Fig. 4a, right panels; Fig. 4c), possibly due to an overriding effect by the osteogenic medium over time.

We also analyzed the ALP expression and activity. ALP is a marker for early osteogenesis. Consistent results on ALP expression and activity were obtained. ALP activity in metformin-treated SHEDs was significantly higher than that of the control cells (Fig. 4d). At day 4, metformin-treated SHEDs showed enhanced ALP staining as compared to the control (Fig. 4e).

\section{Activation Of Ampk By Metformin Treatment In Sheds}

To validate metformin as an AMPK activator in SHEDs, we detected the expression of the AMPK phosphorylated at threonine 172 (p-AMPK Thr172) by immunoblotting. After treatment with metformin $(10,50,100$, and $200 \mu \mathrm{M})$ for $24 \mathrm{~h}$, the expression of p-AMPK (Thr172) was significantly increased as compared to the control treatment (Fig. 5). Interestingly, consistent with the above osteogenic effects by metformin, the activation of AMPK seemed to be most significant at metformin concentration of $100 \mu \mathrm{M}$. A dose-dependent effect on AMPK activation by metformin treatment was observed at clinically relevant concentrations; however, the effect might begin to reduce at a higher concentration of metformin such as $200 \mu \mathrm{M}$ tested in the present study. In the subsequent experiments, $100 \mu \mathrm{M}$ of metformin was employed.

\section{Osteogenic Effects by Metformin in SHEDS via AMPK Signaling}

To determine the role of AMPK signaling in the osteogenic effects by metformin in SHEDs, we added AMPK inhibitor in the cultures. 6-[4-(2-Piperidin-1-ylethoxy) phenyl]-3-pyridin-4-ylpyrazolo [1,5-a]pyrimidine (Compound $\mathrm{C}$ ) is widely used as a cell-permeable AMPK inhibitor [23]. Compound $\mathrm{C}$ treatment dramatically decreased the expression of p-AMPK (Thr172) (Fig. 6a-b). Notably, compound C treatment not only abolished the activation of AMPK by metformin but also reduced the constitutive level of active AMPK in SHEDs. The mineralization enhanced by metformin treatment was fully abolished by Compound C treatment (Fig. 6c-d). Consistent with its inhibition towards constitutive AMPK activation, Compound C treatment alone also led to a decreased mineralization in SHEDs. Furthermore, as compared to metformin-treated cells and vehicle-treated control cells, the expressions of osteogenic genes, including Col I, ALP, RUNX2, and OCN were significantly reduced in the presence of Compound C (Fig. 6e-h). These 
results indicated that AMPK activation was required in the promotion of osteogenic differentiation by metformin in SHEDs.

\section{Discussion}

Mesenchymal stem cells (MSCs) are basal cells that can adhere to grow and express certain signature surface marker proteins such as CD73, CD90, and CD105, but not others such as CD45, CD14, CD34 which express in hematopoietic cells and CD31 which expresses in endothelial cells [24]. Metformin has been widely reported to promote osteogenic differentiation by several types of MSCs, such as those derived from bone marrow, placenta, umbilical cord blood, adipose tissue, and muscle [25-28]. However, while the great clinical potential of SHEDs in tissue engineering has been increasingly recognized, it was unknown whether metformin treatment could induce the osteogenic differentiation of SHEDs. In this study, we have demonstrated that metformin treatment can significantly increase the expression of osteogenic genes and mineralization in SHEDs. Moreover, the osteogenic effect of metformin in SHEDs was found to be via AMPK activation.

Our results firstly confirmed that SHEDs are MSCs. Metformin treatment did not affect the viability and proliferation of our SHEDs at up to $200 \mu \mathrm{M}$, which is in line with the fact that metformin is a safe drug widely used in diabetic patients. However, in a previous report, metformin treatment at $100 \mu \mathrm{M}$ showed moderate cytotoxicity in human chorionic villous MSCs [27]. These different observations may be due to the differences in experimental conditions and/or cell types. Alternatively, there could be subtle cytotoxicity associated with the high concentration of metformin treatment in SHEDs that was undetectable with our assays yet. Actually, the effects of metformin on osteogenic gene expression, mineralization, and AMPK activation were maximized at $100 \mu \mathrm{M}$ in our SHEDs. In clinical patients, the plasma concentration of metformin is around $10 \mu \mathrm{M}$ [29]. Our data indicated that metformin treatment could significantly promote osteogenic differentiation for SHEDs at the low concentrations relevant to these clinical levels. When directly used at a local site such as tissue engineering for specific bone regeneration and repair, a concentration higher than those in the plasma may be employed to achieve optimal effects.

Ischemia is one of the major risk factors for reduced bone healing. Blood vessels not only provide oxygen, but also serve as conduits for additional osteoblasts, which play a positive role in promoting cell differentiation, and are necessary for bone regeneration. Oxygen sensor and hypoxia play an important role in regulating cell entry, vascularization, cell differentiation and bone formation [30]. BMP2 and VEGFA have synergistic effects as osteogenic and angiogenic factors, and they are particularly important for osteogenesis-angiogenesis coupling in MSCs [31,32]. VEGF expressed by osteogenically differentiating MSCs plays a vital role in osteogenesis-angiogenesis coupling during bone regeneration. OPG can competitively inhibit the binding of RANK and RANKL, inhibit osteoclast function, and reduce bone destruction. The ratio of RANKL/OPG is crucial to the balance between bone resorption and bone formation. [22]. However, BMP2, VEGFA, and RANKL/OPG have not been explored in previous studies of metformin in osteogenic differentiation of MSCs. Our results showed that metformin promoted the 
expression of BMP2 and VEGFA, decreased the expression of RANKL/OPG in SHEDs. The results indicate that metformin only promotes osteogenic differentiation of SHEDs and the expression of proangiogenic/osteogenic growth factors, but also inhibits the expression of osteoclastogenic factors.

AMPK is a serine/threonine protein kinase that acts as a sensor for cellular energy and nutrition [33]. The protein complex consists of one catalytic subunit ( $a$ ) and two regulatory subunits ( $\beta$ and $\gamma$ ) [34]. The intracellular AMP/ATP ratio increases in cells subjected to stress or energy depletion. Increased level of AMP triggers allosteric activation of AMPK by binding to AMPKy subunits, leading to exposure of the phosphorylation site at AMPK a Thr172. AMPK a Thr172 can be phosphorylated by Liver kinase B1 (LKB1) and Calcium/calmodulin-dependent protein kinase kinase (CaMKK) [35]. AMPK activation has been reported to promote bone formation and knocking out the $a$, or $\beta$ subunit of AMPK in mice can significantly reduce bone mass[36]. Anti-diabetic metformin suppresses cancer development[37], inhibits adipogenesis, promotes the blood vessel formation, and increases osteogenesis but reduces bone resorption[27, 38, 39]. All of these effects by metformin seem to be related to its action as an AMPK activator. In the present study, we demonstrated that metformin could also increase the activation (i.e., phosphorylation at Thr172) of AMPK in SHEDs. AMPK inhibitor compound C abolished the metformininduced osteogenic differentiation of SHEDs. Our data and abundant literature evidence indicate osteogenic effects of metformin, but Jeyabalan and colleagues reported no effects of metformin on osteogenesis in vivo $[40,41]$. It is likely that the seed cells used in some studies did not have sufficient osteogenic potential or that the animal models were not close enough to the clinical situation of bone regeneration. SHEDs have multiple advantages, such as low immunogenicity, high viability, and abundant clinical sources. Our findings have thus warranted further studies, in particular preclinical evaluation in animals, to explore the clinical potential of SHEDs in the combination with metformin in the areas of bone regeneration and repair.

The molecular mechanism underlying the role of AMPK activation in osteogenic differentiation and bone regeneration remains unclear. AMPK activation may promote bone formation by enhancing autophagy [42]. AMPK controls the osteogenic differentiation of human MSCs through early mTOR inhibitionmediated autophagy and late activation of the Akt/mTOR signaling axis [43].On the other hand, as a direct substrate of AMPK, RUNX2 can stimulate osteoblast differentiation and bone formation [44]. In addition to promoting bone formation, the activation of the AMPK pathway can inhibit bone resorption [45]. Interestingly, we found that metformin can not only promote osteogenesis but also increase the expression of vascular genes and decrease the expression of osteoclast genes in SHEDs. Future mechanistic studies on osteogenic effects of metformin in SHEDs may reveal important insights into osteogenic differentiation of stem cells and bone metabolism.

In conclusion, metformin treatment has no adverse effect on SHEDs and enhances the osteogenic differentiation of SHEDs by activating the AMPK pathway. Metformin upregulates the expression of osteogenic and angiogenic growth factors (BMP2 and VEGF). Interestingly, metformin reduces the expression of osteoclastogenic factors (RANKL/OPG) in SHEDs. Our findings indicate that SHEDs combined with metformin possess great translational potential, especially for bone regeneration and 
bone defect repair. Further studies on the effects of metformin combined with SHEDs on in vivo models of bone defects would be critical to evaluate this potential.

\section{Declarations}

\section{Ethics approval and consent to participate}

The study was approved by the Research Ethics Committee of Guangzhou Medical University (KY2019008) in February 22nd, 2019. The written informed consents were obtained from the parents of the donors of deciduous teeth.

\section{Consent for Publication}

The content of the manuscript has been approved by all the authors.

\section{Availability of data and material}

Some or all data, models, or code generated or used during the study are available from the corresponding author by request.

\section{Competing interests}

The authors declare that they have no conflict interests, either financial or nonfinancial, that could be perceived as prejudicing the impartiality of the research reported.

\section{Funding}

This work was supported by Guangdong Science and Technology Department (2017A020215141) and Guangzhou Science Technology and Innovation Commission (201707010026), China.

\section{Authors' contribution}

$\mathrm{XZ}$, WH: experiment, data analysis and manuscript preparation; JLP, CZ, YL and HG: data analysis, figure preparation and data interpretation; YS, SZ and LG: Study design, data interpretation, manuscript revision; All authors have read and approved the final version of the manuscript. 


\section{Ackonwledgements}

Not applicable

\section{References}

1. Bigham-Sadegh A, Oryan A. Selection of animal models for pre-clinical strategies in evaluating the fracture healing, bone graft substitutes and bone tissue regeneration and engineering. Connect Tissue Res. 2015;56:175-94.

2. Ducy P, Schinke T, Karsenty G. The osteoblast: a sophisticated fibroblast under central surveillance. 289. New York: Science; 2000. pp. 1501-4.

3. Seeman E. Pathogenesis of bone fragility in women and men. Lancet. 2002;359:1841-50.

4. Gay IC, Chen S, MacDougall M. Isolation and characterization of multipotent human periodontal ligament stem cells. Orthod Craniofac Res. 2007;10:149-60.

5. Khojasteh A, Motamedian SR, Rad MR, Shahriari MH, Nadjmi N. Polymeric vs hydroxyapatite-based scaffolds on dental pulp stem cell proliferation and differentiation. World J Stem Cells. 2015;7:1215-21.

6. Akpinar G, Kasap M, Aksoy A, Duruksu G, Gacar G, Karaoz E. Phenotypic and proteomic characteristics of human dental pulp derived mesenchymal stem cells from a natal, an exfoliated deciduous, and an impacted third molar tooth. Stem Cells Int. 2014;2014:1-19.

7. Xie J, Zhao YM, Rao NQ, Wang XT, Fang TJ, Li XX, et al. [Comparative study of differentiation potential of mesenchymal stem cells derived from orofacial system into vascular endothelial cells]. Beijing da xue xue bao Yi xue ban = Journal of Peking University Health sciences. 2019;51:900-6.

8. Hii Siew C, Norhayati L, Ismail Ab R, Kannan Thirumulu P. Expression of Odontogenic and Osteogenic Markers in DPSCs and SHED: A Review. Curr Stem Cell Res Ther. 2017;12:71-9.

9. Ma L, Makino Y, Yamaza H, Akiyama K, Hoshino Y, Song G, et al. Cryopreserved dental pulp tissues of exfoliated deciduous teeth is a feasible stem cell resource for regenerative medicine. PloS one. 2012;7:e51777.

10. Carnevale V, Romagnoli E, D'Erasmo E. Skeletal involvement in patients with diabetes mellitus. Diab/Metab Res Rev. 2004;20:196-204.

11. Vestergaard P, Rejnmark L, Mosekilde L. Relative fracture risk in patients with diabetes mellitus, and the impact of insulin and oral antidiabetic medication on relative fracture risk. Diabetologia. 2005;48:1292-9.

12. Tan MH, Alquraini H, Mizokami-Stout K, MacEachern M. Metformin: From Research to Clinical Practice. Endocrinol Metab Clin North Am. 2016;45:819-43.

13. Bailey CD. C. Metformin: its botanical background. Pract Diab Int. 2004;21:115-7. 
14. Orciani M, Fini M, Di Primio R, Mattioli-Belmonte M. Biofabrication and Bone Tissue Regeneration: Cell Source, Approaches, and Challenges. Front Bioeng Biotechnol. 2017;5:17.

15. Melton LJ 3rd, Leibson CL, Achenbach SJ, Therneau TM, Khosla S. Fracture risk in type 2 diabetes: update of a population-based study. J Bone Miner Res. 2008;23:1334-42.

16. Shu Y, Sheardown SA, Brown C, Owen RP, Zhang S, Castro RA, et al. Effect of genetic variation in the organic cation transporter 1 (OCT1) on metformin action. J Clin Invest. 2007;117:1422-31.

17. Aljofi F. Metformin and Mesenchymal Stem Cell Osteogenic Differentiation: Role of Organic Cation Transporters. Dissertation,. 2017; University of Maryland.

18. Hardie DG, Ross FA, Hawley SA. AMPK: a nutrient and energy sensor that maintains energy homeostasis. Nature reviews Molecular cell biology. 2012;13:251-62.

19. Claesson-Welsh L, Welsh M. VEGFA and tumour angiogenesis. J Intern Med. 2013;273:114-27.

20. Salazar VS, Gamer LW, Rosen V. BMP signalling in skeletal development, disease and repair. Nature reviews Endocrinology. 2016;12:203-21.

21. El-Rifai O, Chow J, Lacombe J, Julien C, Faubert D, Susan-Resiga D, et al. Proprotein convertase furin regulates osteocalcin and bone endocrine function. Journal of Clinical Investigation. 2017;127.

22. Liu C, Walter TS, Huang P, Zhang S, Zhu $X$, Wu Y, et al. Structural and functional insights of RANKLRANK interaction and signaling. Journal of immunology (Baltimore, Md: 1950). 2010;184:6910-9.

23. Liu X, Chhipa R, Nakano I, Dasgupta B. The AMPK inhibitor Compound C is a potent AMPKindependent antiglioma agent. Molecular cancer therapeutics. 2014;13.

24. Dominici M, Le Blanc K, Mueller I, Slaper-Cortenbach I, Marini FC, Krause DS, et al. Minimal criteria for defining multipotent mesenchymal stromal cells. The International Society for Cellular Therapy position statement. Cytotherapy. 2006;8:315-7.

25. Marycz K, Tomaszewski KA, Kornicka K, Henry BM, Wronski S, Tarasiuk J, et al. Metformin Decreases Reactive Oxygen Species, Enhances Osteogenic Properties of Adipose-Derived Multipotent Mesenchymal Stem Cells In Vitro, and Increases Bone Density In Vivo. Oxidative Med Cell Longev. 2016;2016:9785890.

26. Al Jofi FE, Ma T, Guo D, Schneider MP, Shu Y, Xu HHK, et al. Functional organic cation transporters mediate osteogenic response to metformin in human umbilical cord mesenchymal stromal cells. Cytotherapy. 2018;20:650-9.

27. Gu Q, Gu Y, Yang H, Shi Q. Metformin Enhances Osteogenesis and Suppresses Adipogenesis of Human Chorionic Villous Mesenchymal Stem Cells. Tohoku J Exp Med. 2017;241:13-9.

28. Wen-Qi Ma X-JS, Wang Y, Zhu Y, Han X-Q, Liu N-F. Restoring mitochondrial biogenesis with metformin attenuates $\beta$-GP-T induced phenotypic transformation of VSMCs into an osteogenic phenotype via inhibition of PDK4/oxidative stress-mediated apoptosis. Mol Cell Endocrinol. 2019;479:39-53.

29. Smolders EJ, Colbers A, de Kanter C, Velthoven-Graafland K, Wolberink LT, van Ewijk-Beneken Kolmer $\mathrm{N}$, et al. Metformin and daclatasvir: absence of a pharmacokinetic-pharmacodynamic drug interaction in healthy volunteers. Br J Clin Pharmacol. 2017;83:2225-34. 
30. Hankenson KG, K \& Shaughnessy M. Extracellular Signaling Molecules to Promote Fracture Healing and Bone Regeneration. Adv Drug Deliv Rev. 2015;94:3-12.

31. Xiao C, Zhou H, Liu G, Zhang P, Fu Y, Gu P, et al. Bone marrow stromal cells with a combined expression of BMP-2 and VEGF-165 enhanced bone regeneration. Biomedical materials (Bristol England). 2011;6:015013.

32. Hu K, Olsen BR. Osteoblast-derived VEGF regulates osteoblast differentiation and bone formation during bone repair. J Clin Invest. 2016;126:509-26.

33. Hardie DG. AMP-activated/SNF1 protein kinases: conserved guardians of cellular energy. Nat Rev Mol Cell Biol. 2007;8:774-85.

34. Hardie DG, Carling D, Carlson M. The AMP-activated/SNF1 protein kinase subfamily: metabolic sensors of the eukaryotic cell? Annu Rev Biochem. 1998;67:821-55.

35. Faubert B, Vincent EE, Poffenberger MC, Jones RG. The AMP-activated protein kinase (AMPK) and cancer: many faces of a metabolic regulator. Cancer Lett. 2015;356:165-70.

36. Kanazawa I, Takeno A, Tanaka KI, Notsu M, Sugimoto T. Osteoblast AMP-Activated Protein Kinase Regulates Postnatal Skeletal Development in Male Mice. Endocrinology. 2018;159:597-608.

37. Pierotti MA, Berrino F, Gariboldi M, Melani C, Mogavero A, Negri T, et al. Targeting metabolism for cancer treatment and prevention: metformin, an old drug with multi-faceted effects. Oncogene. 2013;32:1475-87.

38. Martin MJ, Hayward R, Viros A, Marais R. Metformin accelerates the growth of BRAF V600E-driven melanoma by upregulating VEGF-A. Cancer discovery. 2012;2:344-55.

39. Gao Y, Li YF, Xue J, Jia YQA, Hu J. Effect of the anti-diabetic drug metformin on bone mass in ovariectomized rats. Eur J Pharmacol. 2010;635:231-6.

40. Lin H, Ying Y, Wang YY, Wang G, Jiang SS, Huang D, et al. AMPK downregulates ALK2 via increasing the interaction between Smurfl and Smad6, leading to inhibition of osteogenic differentiation. BbaMol Cell Res. 2017;1864:2369-77.

41. Jeyabalan J, Viollet B, Smitham P, Ellis SA, Zaman G, Bardin C, et al. The anti-diabetic drug metformin does not affect bone mass in vivo or fracture healing. Osteoporosis Int. 2013;24:265970.

42. Li Y, Su JJ, Sun WC, Cai L, Deng ZM. AMP-activated protein kinase stimulates osteoblast differentiation and mineralization through autophagy induction. Int J Mol Med. 2018;41:2535-44.

43. Aleksandar Pantovic AK, Kristina Janjetovic J, Kocic L, Harhaji-Trajkovic D, Bugarski, Vladimir Trajkovic. Coordinated time-dependent modulation of AMPK/Akt/mTOR signaling and autophagy controls osteogenic differentiation of human mesenchymal stem cells. Bone. 2013;52:524-31.

44. Chava S, Chennakesavulu S, Gayatri BM, Reddy ABM. A novel phosphorylation by AMP-activated kinase regulates RUNX2 from ubiquitination in osteogenesis over adipogenesis. Cell Death Dis. 2018;9:754. 
45. Yamaguchi N, Kukita T, Li YJ, Kamio N, Fukumoto S, Nonaka K, et al. Adiponectin inhibits induction of TNF-alpha/RANKL-stimulated NFATc1 via the AMPK signaling. FEBS Lett. 2008;582:451-6.

\section{Figures}
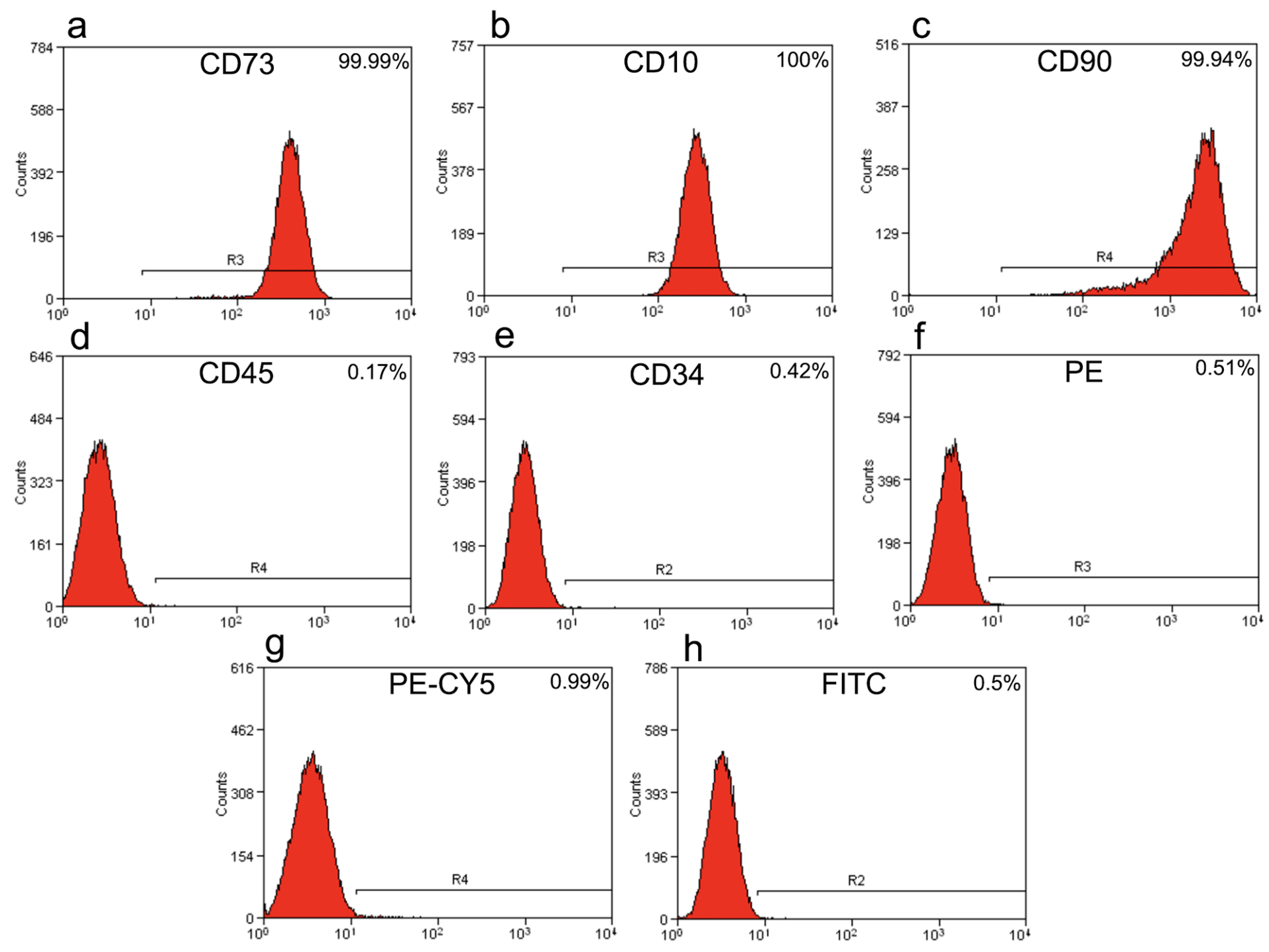

\section{Figure 1}

Detection of cell surface marker antigens in SHEDs by flow cytometry. Flow cytometry was used to detect molecular surface antigen markers. As shown, CD45 and CD34 were negative, while CD73, CD105 and CD90 were positive. 

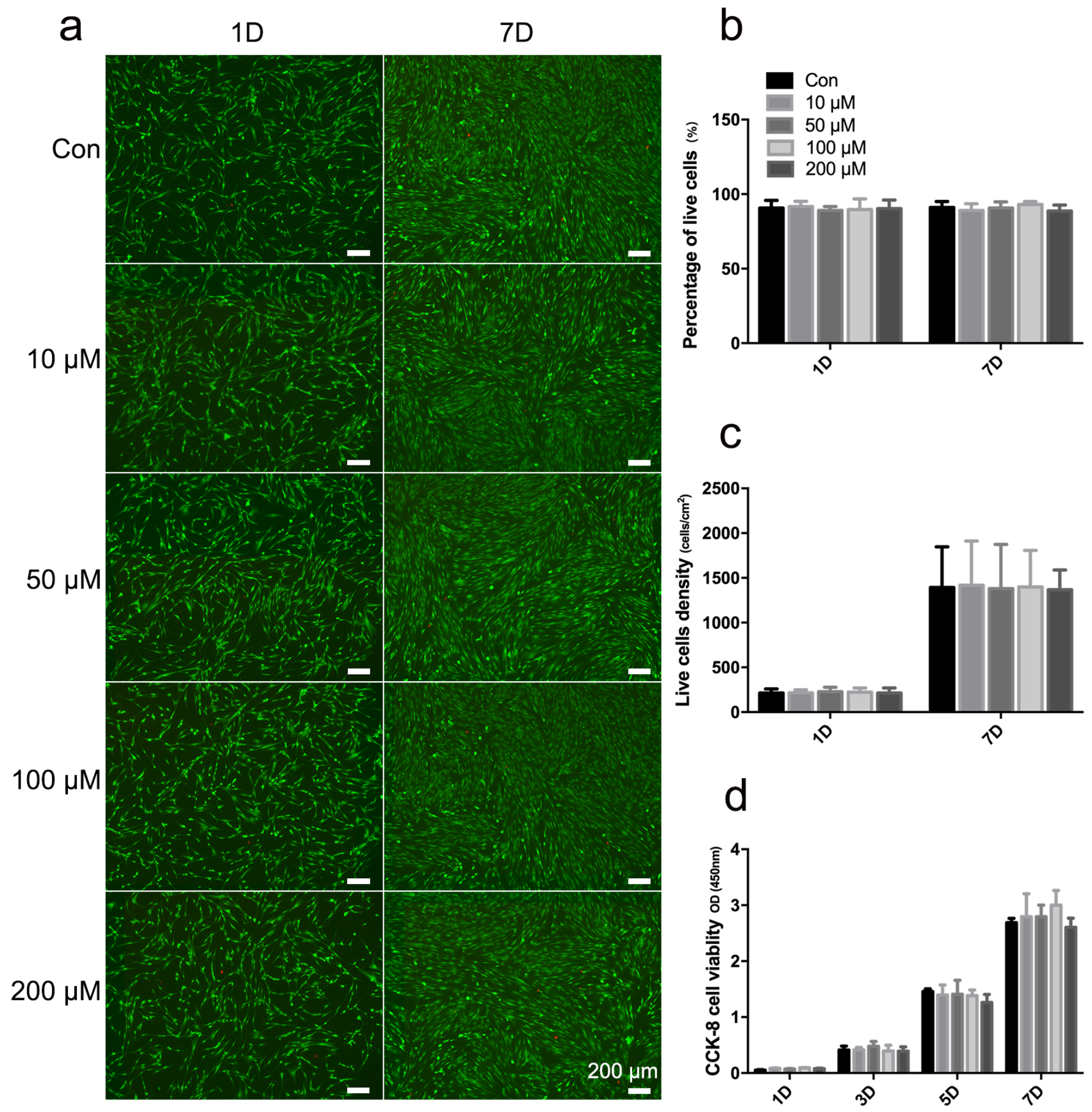

Figure 2

Effect of metformin treatment on SHED viability. SHEDs were stained as live and dead cells (a) on day 1 and 7. Green, living cell; Red, dead cells. The percentage of live cells was quantitated under an inverted fluorescence microscopy (b). The cell density of SHEDs was higher on day 7 than that on day 1 ; however, there were no differences between metformin-treated and control cells (c). The results from cck-8 assay also indicated that metformin treatment $(0-200 \mu \mathrm{M})$ did not affect the proliferation of SHEDs over time from day 1 to day 7 (d). 

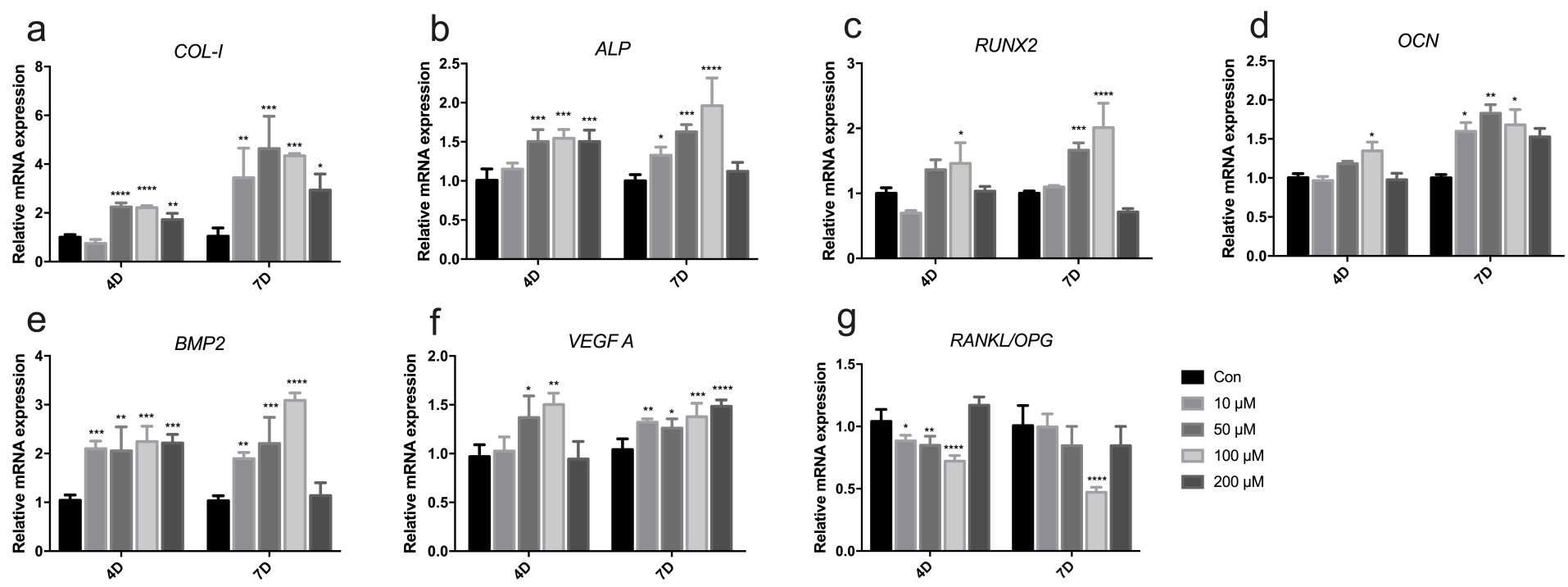

Figure 3

Effect of metformin treatment on osteogenic gene expression in SHEDs. P4 SHEDs were treated with the indicated concentrations of metformin for 4 or 7 days. The expression of genes (OCN, COL-I, RUNX2, ALP,

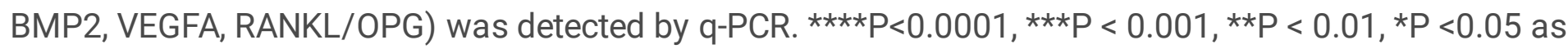
compared to the control. 


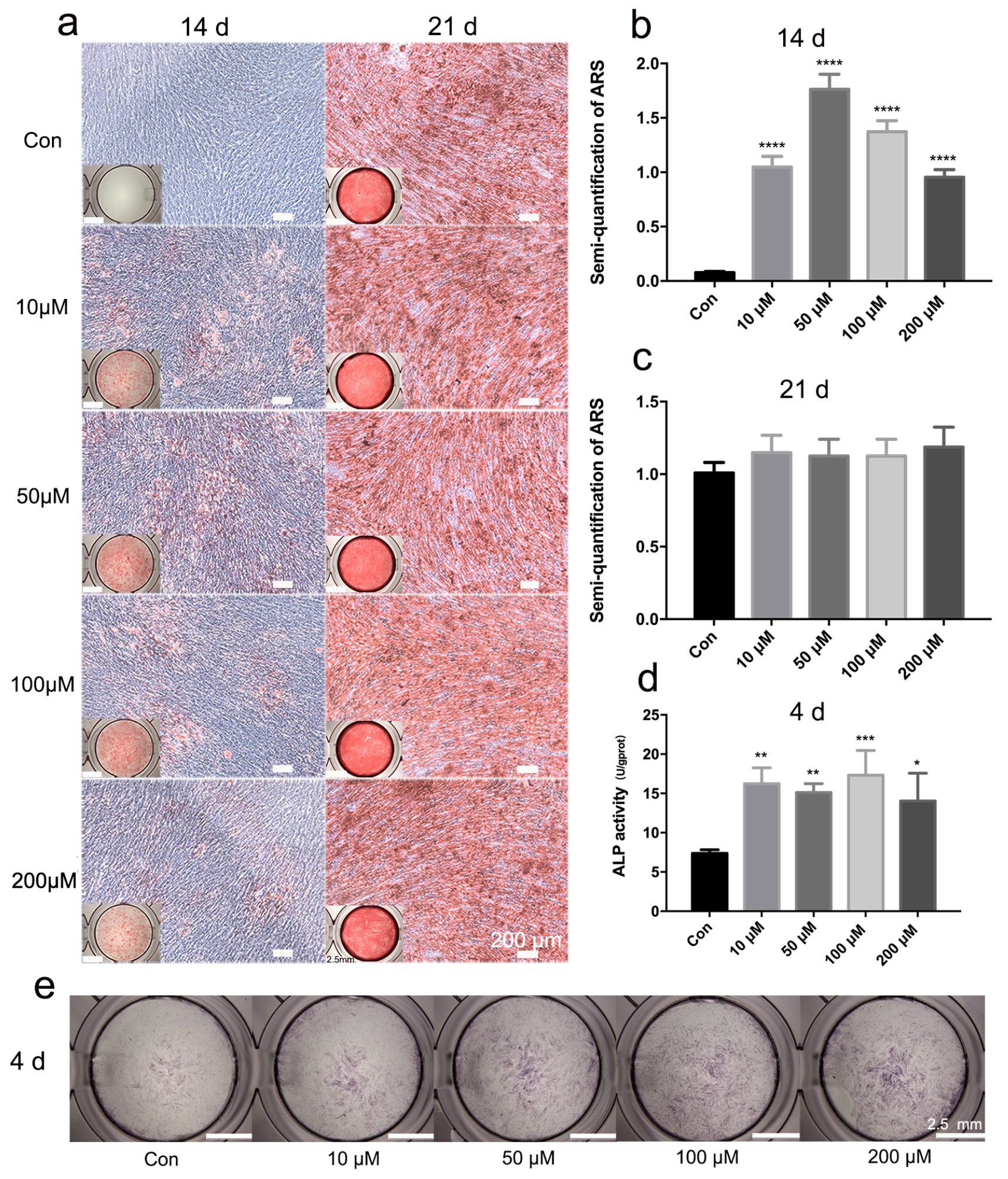

Figure 4

Effect of metformin treatment on osteogenic differentiation of SHEDs. SHEDs were cultured in osteogenic induction medium for 14 or 21 days. The degree of mineralization was assessed by ARS (scale bar $=2.5 \mathrm{~mm}$ ). (a). The Semi-quantitative result of mineralized matrix $(b-c)$. SHEDs were treated with the indicated concentrations of metformin for 4 day. (d) ALP activity and (e) ALP staining of SHEDs. ${ }^{* \star * *} \mathrm{P}<0.0001,{ }^{* * *} \mathrm{P}<0.001,{ }^{* *} \mathrm{P}<0.01,{ }^{*} \mathrm{P}<0.05$ as compared to the control. 
a

b

Met $(\mu \mathrm{M}) \quad 0 \quad 10 \quad 50 \quad 100 \quad 200$
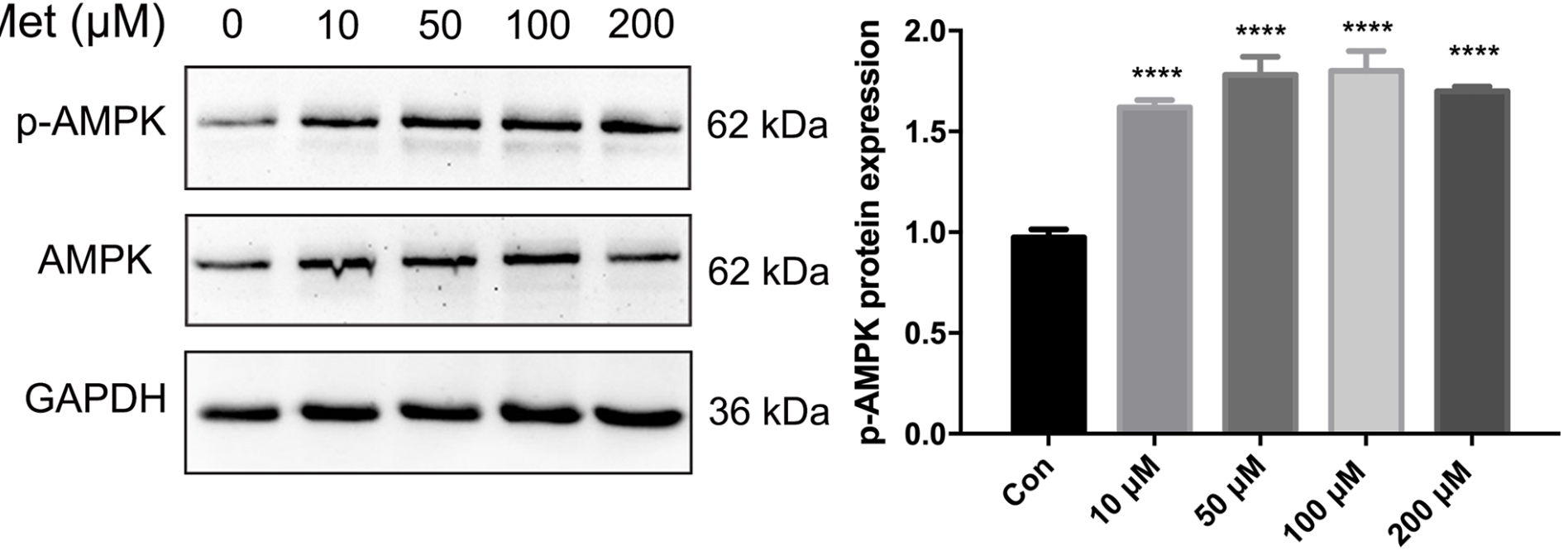

Figure 5

Effect of metformin treatment on AMPK activation in SHEDs. SHEDs were treated with the indi-cated concentrations of metformin for $24 \mathrm{~h}$. The expression of AMPK a Thr172 was detected by western blot. $\star \star \star \star * P<0.0001$ as compared to the control. 
Comp.C $(\mu \mathrm{M}) \quad-\quad 10 \quad 10 \quad-\quad$ C
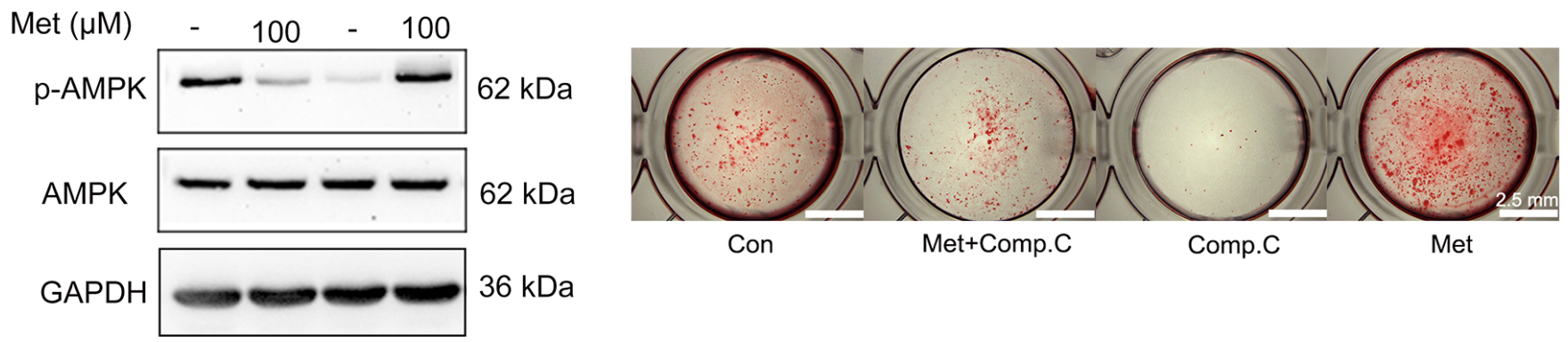

b
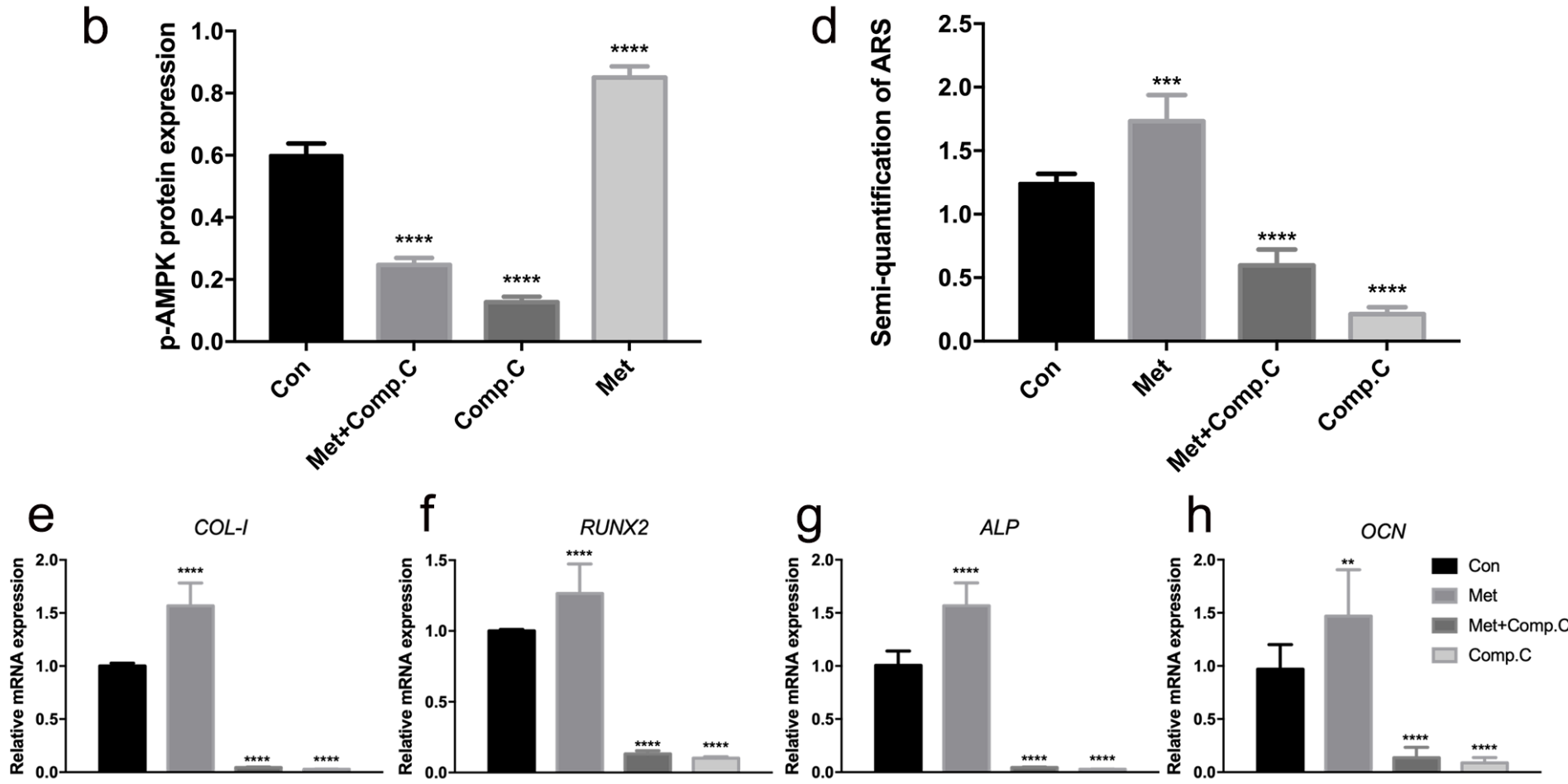

Figure 6

AMPK activation was required in the promotion of osteogenic differentiation by metformin in SHEDs. SHEDs were treated with the AMPK inhibitor Compound C $(10 \mu \mathrm{M})$ and/or metformin $\otimes 100 \mu \mathrm{M} \otimes$ for $24 \mathrm{~h}$. The expression of AMPK a Thr172 was detected by western blot (a-b). SHEDs were cul-tured in osteogenic induction medium for 10 days. Mineralized nodule was assessed by ARS (scale bar $=2.5$ $\mathrm{mm}$ ). (c-d). SHEDs were treated with Compound C and/or metformin for 4 days. The expression of osteogenic genes was detected by $q-P C R(e-h)$. The data were shown as means \pm SD. ${ }^{*} \star \star * P<0.0001$, ${ }^{\star} * \star P<0.001$ as compared to the control. Con, control; Met+Comp.C, metformin + Compound C; Comp.C, Compound C; Met, metformin.

\section{Supplementary Files}

This is a list of supplementary files associated with this preprint. Click to download.

- Graphicalabstract.tif 